\title{
Curve It, Gauge It, or Leave It? Practical Underdetermination in Gravitational Theories
}

\author{
Holger LyRe \\ Institut für Philosophie, Ruhr-Universität Bochum, D-44780 Bochum, Germany, \\ Email: holger.lyre@ruhr-uni-bochum.de
}

\section{Tim Oliver Eynck}

NIKHEF, Kruislaan 409, Postbus 41882, NL-1009 DB Amsterdam, The Netherlands, Email: teynck@nikhef.nl

December 2001

\begin{abstract}
Four empirically equivalent versions of general relativity, namely standard GR, LORENTZ-invariant gravitational theory, and the gravitational gauge theories of the LORENTZ and translation groups, are investigated in the form of a case study for theory underdetermination. The various ontological indeterminacies (both underdetermination and inscrutability of reference) inherent in gravitational theories are analyzed in a detailed comparative study. The concept of practical underdetermination is proposed, followed by a discussion of its adequacy to describe scientific progress.
\end{abstract}

\section{Content}

1 Introduction $\quad 2$

2 Equivalent Descriptions of Gravity $\quad 3$

2.1 General Relativity . . . . . . . . . . . . . . . . . . . . . . 3

2.2 Lorentz-invariant Gravitation . . . . . . . . . . . . . . . . . . . . . . . 4

2.3 Gravity as a Gauge Theory? . . . . . . . . . . . . . . . . . . . . 5

2.3.1 Yang-Mills Gauge Theories . . . . . . . . . . . . . . . 5

2.3 .2 Heuristic Gauging . . . . . . . . . . . . . . . . . . . 7

2.3 .3 Poincaré Gauge Theory . . . . . . . . . . . . . . . . . 8

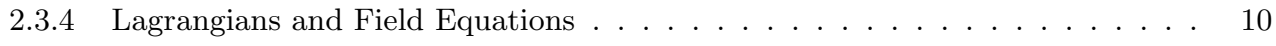

2.4 Curve It, Gauge It, or Leave It? . . . . . . . . . . . . . . . . . . . . . . . . . . . . . 12

3 Indeterminacies in Gravitational Theories 12

3.1 Theories, Models, and the Issue of Underdetermination . . . . . . . . . . . . . . 13

3.2 Ontological Difference-Topological Indifference . . . . . . . . . . . . . . . . . . . . . 14

3.3 Inscrutability of Reference: The Habitat of the Gravitational Field . . . . . . . . . . 15

3.4 Inscrutability of Reference: Curvature versus Torsion . . . . . . . . . . . . . . . . 15 


\section{Introduction}

Asked to judge the impact of physics achievements during the 20th century one would probably be inclined to ascribe particular importance to the concept of gauge field theories. However, they have become an object of study for philosophers of science only recently. AuYANG (1995) can be regarded as marking this change. Over the past years gauge theories have enjoyed a certain increase in popularity within the philosophy of science community, and were even considered worthy of a dedicated session at the recent PSA 2000 conference (cf. EARMAN 2001).

Albeit having received little attention so far from philosophers, and even hardly known to many physicists, general relativity can be cast in formulations bearing great similarities to gauge theories. Such approaches have up to now been discussed mainly in the context of quantum gravity and its interpretational dilemmas (cf. e.g. CAllender and HuggetT 2001). Guided by the conceptional and technical difficulties arising in the canonical quantization of constrained systems, these studies concentrate on aspects of the Hamiltonian approach (cf. again EARMAN 2001). However, for studying the YANG-Mills theories of the standard model Lagrangian methods are at least equally important, but so far only few studies in the philosophy of science have dealt with them (LIU 2001 is a notable exception). In the present work we will be focussing on YANG-MILLS-like gauge theoretic formulations of gravity.

Our study of four such formulations empirically equivalent (in a sense to be made precise later) to orthodox general relativity leads us naturally to the question of theory underdetermination by empirical data, a classical philosophy of science topic. The thesis of theory underdetermination by a given body of empirical evidence roots in the claim that any scientific theory unavoidably contains more than only pure observational terms. It therefore features in its explanatory apparatus theoretical terms which, since they refer to non-observable structures, are open to metatheoretic dispute.

Although the underdetermination thesis is usually stated in a rather abstract fashion, we deem it of particular importance to consider concrete examples. We believe that only the indisputable existence of such would permit a final verdict. Establishing connections between the terminology of the underdetermination discussion on the one hand and current scientific research on the other therefore seems to us highly desirable. As a step in this direction we present in this paper a case study of four equivalent theories of gravity, viz

$T_{1}$ : standard general relativity in RIEMANNian spacetime (standard GR),

$T_{2}$ : LoREnTz-invariant gravitational theory in flat spacetime (flat GR),

$T_{3}$ : gauge theory of the LORENTz group (rotational GT), and

$T_{4}$ : gauge theory of the translation group (translational GT).

Clearly, alternative formulations of GR constitute - to theoretical physicists as well as to philosophers of science - an interesting subject in its own right. Especially the second of these groups our work might serve as a starting point, in particular for the many references given to the relevant literature.

The present article is structured roughly as follows. Since we are investigating and comparing non-standard physical concepts we need to discuss the underlying mathematical framework in 
quite some detail in section 2. We summarize standard GR only very briefly, but since gauge theories of gravity are almost unknown to philosophers of science - they seem to receive scant attention only in the physics community - we felt that the somewhat lengthy and in places technical introduction to these, i.e. in our enumeration theories $T_{3}$ and $T_{4}$, justified an almost philosophy-devoid section of its own. The conceptual analysis of the four theories presented is mainly confined to sections 3 and 4 . We will try and place our examples in the broader context of ontological indeterminacies such as inscrutability of reference and theory underdetermination (section 3). As a special result we propose the concept of practical underdetermination (section 4) which - as we believe - is ideally suited to capture besides (static) scientific edifices also the nature of its progress.

\section{Equivalent Descriptions of Gravity}

In this section we will describe in as much detail as necessary for the case study of this paper the physics of the theories $T_{1}$ up to $T_{4}$ as introduced in the previous section. We assume that the reader is familiar with the basics of (quantum) field theory and standard GR, which is why we can be rather brief in subsection 2.1. This is followed by another short subsection 2.2 introducing $T_{2}$, a LoRentz-invariant gravitational theory in flat spacetime. Differing in their concepts of curvature as they may, both these theories stand apart from the rather unified framework describing the other three fundamental forces known today. This standard model of elementary particles is, unlike the gravitational theories $T_{1}$ and $T_{2}$, based on the gauge principle. A synopsis of particle physics and the mathematical apparatus it employs would go far beyond the scope of this study, but we do remind the reader of one of its core concepts, namely YANG-MILLS theories (section 2.3.1). There exist treatments of the gravitational interaction which unlike GR follow rather closely these gauge ideas as employed in particle physics. Typically these alternative formulations go beyond Einstein's theory in predicting corrections, but at least two of them, namely cases $T_{3}$ and $T_{4}$, are, at least in the limit of spinless matter, equivalent to it. Not in any sense attempting a comprehensive summary of all these approaches we focus in the nevertheless rather lengthy and technical subsection 2.3 on their aspects important for our study. We then conclude this preparatory section with a short summary which leads us to the core of our study, namely sections 3 and 4 .

\subsection{General Relativity}

General relativity (GR), the theory commonly accepted to describe gravitational interactions, is formulated in the language of RIEMANNian geometry. It is even today still presented in much the same fashion as originally proposed by Einstein. Whereas in the standard model of elementary particles the fundamental entities are quantum fields carrying spin, in GR this role is played by spinless point matter which follows geodesic trajectories. In the absence of gravitational fields these are described by the geodesic equation of SR

$$
\frac{d}{d \tau} v^{\mu}(\tau)=0
$$


with four-velocity $v^{\mu}$. If the flat MINKOWSKI metric $\eta_{\mu \nu}$ is replaced by a function of the coordinates $g_{\mu \nu}(x)$, geodesic trajectories on (curved) spacetime are prescribed by

$$
\frac{d}{d \tau} v^{\mu}(\tau)+\{\}_{\nu \rho}^{\mu} v^{\nu}(\tau) v^{\rho}(\tau)=0,
$$

where the CHRISTOFFEL symbols of the connection are derived from the metric according to

$$
\{\}_{\mu \nu}^{\lambda}=\frac{1}{2} g^{\lambda \sigma}\left(\partial_{\mu} g_{\sigma \nu}+\partial_{\nu} g_{\sigma \mu}-\partial_{\sigma} g_{\mu \nu}\right) .
$$

Under coordinate transformations $x \longrightarrow x^{\prime}$ the connection transforms inhomogeneously as

$$
\{\}_{\mu \nu}^{\lambda} \rightarrow\{\}_{\mu \nu}^{\prime \lambda}=\frac{\partial x^{\prime \lambda}}{\partial x^{\rho}} \frac{\partial x^{\sigma}}{\partial x^{\prime \mu}} \frac{\partial x^{\tau}}{\partial x^{\prime \nu}}\{\}_{\sigma \tau}^{\rho}+\frac{\partial x^{\prime \lambda}}{\partial x^{\rho}} \frac{\partial^{2} x^{\rho}}{\partial x^{\prime \mu} \partial x^{\prime \nu}},
$$

whereas the RIEMANN tensor or curvature

$$
R_{\lambda \mu \nu}^{\kappa}=\partial_{\mu}\{\}_{\lambda \nu}^{\kappa}-\partial_{\nu}\{\}_{\lambda \mu}^{\kappa}+\{\}_{\lambda \nu}^{\rho}\{\}_{\rho \mu}^{\kappa}-\{\}_{\lambda \mu}^{\rho}\{\}_{\rho \nu}^{\kappa}
$$

as well as the RICCI tensor $R_{\mu \nu}=R_{\mu \lambda \nu}^{\lambda}$ and the RICCI scalar $R=R_{\mu}^{\mu}$ all transform homogeneously. They can thus be used in the Einstein-HiLBERT Lagrangian

$$
\mathcal{L}_{G R} \sim \sqrt{-g} R
$$

where $g$ is the determinant of the metric, leading to the EINSTEIN equations, the equations of motion for GR's dynamic field $g_{\mu \nu}(x)$. The coupling of matter to the gravitational field is motivated by the principle of minimal substitution, involving the uniquely determined LEVICivita connection \{\} . Hence the transition from SR to GR basically amounts to replacing partial by covariant derivatives so as to ensure that by a clever choice of coordinates (in GR parlance the freely falling elevator) the effects of gravity can always be transformed away at least at a particular point. Different views have been taken in the literature as to whether the metric itself or the curvature as formed from second derivatives of the former are to be regarded primary. However, this issue will not need to concern us in the following.

\subsection{Lorentz-invariant Gravitation}

Having sketched GR as our first gravitational theory, $T_{1}$, we shall now consider the LORENTZinvariant gravitational theory $T_{2}$. It intends to represent the gravitational in as close a fashion as possible to the electromagnetic field, taking seriously the special role of Minkowski space only implicit in usual GR. This so to speak flat GR, as first developped by GUPTA $(1952,1957)$ and Thirring $(1959,1961),{ }^{1}$ considers a dynamic field $h_{\mu \nu}(x)$ against a flat Minkowski background metric, viz.

$$
g_{\mu \nu}=\eta_{\mu \nu}+\kappa h_{\mu \nu}
$$

This expression for the metric tensor leads at the level of the Lagrangian to a power series expansion in $\kappa$. By going to high enough order in the coupling $\kappa$, flat GR can approximate standard GR to any accuracy desired (the details can be found in the references given abovehere we are just interested in the conceptual framework). $T_{2}$ is thus empirically equivalent to $T_{1}$,

${ }^{1}$ Cf. also Barbour (1967) and Mittelstaedt and Barbour (1967). 
which was already termed a "philosophical" result in Gupta 1957. Except possibly for global effects, no experiment can distinguish between the two theories. If GR is viewed as a local field theory only, but not as supposed to describe the universe in toto, such global, cosmological effects move outside its realm and need not be worried about. We will come back to this point in more detail in the course of section 3 .

\subsection{Gravity as a Gauge Theory?}

We will now introduce the reader to the concepts of theories $T_{3}$ and $T_{4}$. They are both based on the gauge principle as employed in the YANG-MILLs theories of the standard model (section 2.3.1.) Contrary to this, gravitational gauge theories involve gauging not internal (charge, colour) but external (rotation, translation) degrees of freedom. We illustrate the difficulties caused by this in section 2.3.2, where we attempt to naively apply the gauge idea to the geodesic equation for a point particle. A mathematically consistent treatment in the framework of field theory requires the introduction of tetrads in section 2.3.3, where we also briefly mention different possible choices of gauge groups, but consider in the end only the particular example of the Poincaré group. The most general Lagrangian possible for this theory is discussed in subsection 2.3.4 and special emphasis put on two particular examples equivalent to standard GR for point matter.

\subsubsection{Yang-Mills Gauge Theories}

In this section we shall rewiew - as far as necessary - the mathematical background of YANGMills gauge theories, and the related fibre bundle approach. The framework of YANG-MiLls gauge theories provides a powerful tool in modern theoretical physics with regard to a unification of the three non-gravitational interactions. One usually starts with a free matter-field theory, such as the DiRAC Lagrangian

$$
\mathcal{L}_{D}=\bar{\Psi}(x)\left(i \gamma^{\mu} \partial_{\mu}-m\right) \Psi(x)
$$

It is in this form clearly invariant under global (i.e. rigid) $S U(n)$ gauge transformations. For the construction of an $S U(n)$ gauge theory we demand covariance of the free matter-field theory under local (i.e. spacetime-dependent) transformations

$$
\Psi(x) \rightarrow \Psi^{\prime}(x)=e^{i g \Lambda^{a}(x) \hat{t}^{a}} \Psi(x)=\hat{U}(x) \Psi(x),
$$

where $\hat{t}^{a}$ are the generators of the corresponding $\mathfrak{s} u(N)$ algebra. This requirement is sometimes called the gauge postulate. The idea is to introduce a vector potential $B_{\mu}^{a}(x)$ with an inhomogeneous $S U(n)$ transformation behaviour

$$
B_{\mu}^{a}(x) \hat{t}^{a} \rightarrow B_{\mu}^{a}(x) \hat{t}^{a}=\hat{U}(x) B_{\mu}^{a}(x) \hat{t}^{a} \hat{U}^{-1}(x)+\hat{U}(x) \partial_{\mu} \hat{U}^{-1}(x)
$$

so that in the DIRAC Lagrangian the partial can be replaced by the so-called covariant derivative

$$
\partial_{\mu} \rightarrow D_{\mu}=\partial_{\mu}+i g B_{\mu}^{a} \hat{t}^{a}
$$

effecting a cancellation of the extra derivative terms and thereby leading to a covariant Lagrangian. In other words, it looks as though - in order to satisfy the postulate of local gauge 
covariance of the free theory - one is forced to introduce a gauge field $B_{\mu}^{a}$ which couples minimally to the matter-field $\psi$. This general strategy to "derive" the interaction from a local symmetry requirement is referred to as the gauge principle. ${ }^{2}$

The sufficiency of this principle alone to justify the introduction of physically relevant interaction fields has recently been questioned. ${ }^{3}$ However, these criticisms, important as they may be in general to help clarify differences and similarities in the interpretational discourse of quantum field theory on the one and spacetime theories (including gravity) on the other hand, are of no importance to the present study, since they are not intended to raise doubts about the general importance of gauge theories, but merely on how they are usually presented.

From the requirement of local gauge invariance we may construct a field strength tensor

$$
F_{\mu \nu}^{a}=\partial_{\mu} B_{\nu}^{a}-\partial_{\nu} B_{\mu}^{a}-g f^{a b c} B_{\mu}^{b} B_{\nu}^{c}
$$

with $f^{a b c}$ the structure constants of the group under consideration, and the corresponding gaugefield Lagrangian

$$
\mathcal{L}_{G F}=-\frac{1}{4} F_{\mu \nu}^{a} F^{a \mu \nu} .
$$

Note that this is the only kinetic term for the gauge fields allowed by gauge covariance of the theory. Thus, we obtain the fully-fledged Lagrangian of YANG-MiLLS theories $\mathcal{L}_{Y M T}=$ $\mathcal{L}_{D}+\mathcal{L}_{\text {int }}+\mathcal{L}_{G F}$. We note here in passing only that one could equally well have started with the Lagrangian of a spinless field instead of the DIRAC spinor considered here. The gauge part of the the full theory, namely the covariant derivative and the gauge field Lagrangian, remains unaltered.

The appropriate mathematical framework to describe the objects introduced in the previous paragraphs is furnished by the theory of fibre bundles. Such fibre bundles consist of a base space and a fibre attached to it at every point. Locally this construction reduces to a Cartesian product of the two spaces involved. Globally there can occur topological effects which, however, will not concern us in the following. If one pictures spacetime as the horizontal and the fibres as the vertical directions in the total space jointly formed by the two, gauge transformations can

\footnotetext{
${ }^{2}$ The general idea was first introduced by HeRmann Weyl in his seminal 1929 paper. See O'RAIFEARTAigh (1995) and O'RAifeartaigh and Straumann (2000) for historical remarks.

${ }^{3}$ Differing as they may in details, all these criticisms pertain to the fact that local gauge transformations correspond to a mere change in the position representation of the wavefunction. The covariant derivative (11) also results from this change. In the fibre bundle language (see below), the occuring gauge connection $B_{\mu}^{a}$ must be considered flat. That is to say the underlying gauge field strength $F_{\mu \nu}^{a}$, given by the derivative of the potential and representing the true interaction force, remains zero. This may best be seen in case of the covariant derivative in gravitational theories - the case which will be considered in detail in section 2.3. There we may have non-vanishing CHRISTOFFEL symbols, but this does not necessarily imply non-vanishing curvature, namely a true gravitational field. Instead, non-vanishing CHRISTOFfEL symbols may occur due to some special choice of coordinates (e.g. curvelinear coordinates in flat space, cf. also the remarks in LIU (2001)). The gauge principle therefore merely gives the form of the minimal interaction coupling term. The introduction of a true interaction field strength must be put in by hand and may be justified by the assumption of a generalized equivalence principle (LyRE 2000). Moreover, due to NoETHER's first theorem, global gauge invariance of the Lagrangian under $S U(n)$ transformations is connected with the existence of $n^{2}-1$ conserved currents $j_{\mu}^{a}(x)=g \bar{\Psi}(x) \gamma_{\mu} \hat{t}^{a} \Psi(x)$ obeying a continuity equation and receiving (for the non-abelian case) contributions also from the (charged) gauge potentials. Hence, in gauge theories we are dealing with at least three principles, namely the conservation principle, the gauge principle and the generalized equivalence principle (LYRE 2001).
} 
be pictured as transformations acting vertically only, i.e. affecting as physicist would call them, internal rotations. They leave the physics unaltered.

Gauge potentials can be interpreted as LIE algebra-valued connections on the bundle. They couple only to fields transforming non-trivially under the gauge group considered. Their corresping bundle curvatures are the physical field strenghts. The fibre bundle formalism will enable us in section 2.4 to state clearly the novel features of gravitational (gauge) theories as opposed to standard quantum field theories.

\subsubsection{Heuristic Gauging}

There exist treatments of the gravitational interaction which follow much more closely the gauge ideas outlined in the previous section. Some of these alternative formulations go beyond GR in predicting new forms of gravitational interactions but at least one is (in a sense to be clarified below) equivalent to EINSTEIN's theory. But before we treat these non-standard approaches in sections 2.3.3 and 2.3.4 at the level of mathematical rigour required for the interpretational sections 3 and 4 we first give a short, somewhat heuristic introduction. It should help to convince the reader unfamiliar with these approaches that from the naive application of a gauge principle to the motion of a point particle there naturally arise mathematical structures very similar to those in GR. Readers familiar with gravitational gauge concepts might therefore want to skip this subsection.

We first observe that GR as presented in section 2.1 as well as the YANG-MiLLS theories reviewed in section 2.3.1 contain (bundle) connections ( \{\}$_{\mu \nu}^{\rho}$ and $B_{\mu}^{a}$, respectively) and (bundle) curvature fields. Their introductions into the theory, however, are motivated differently. Could not gravity also be derived following some kind of gauge principle? Consider again the equation of motion of a free test particle in MinkOwski space with four-velocity $v^{\mu}$ parameterized by its proper time $\tau$ such that $\frac{d v^{\mu}(\tau)}{d \tau}=0$. In SR this expression is invariant under (rigid) LoRENTz rotations. In the spirit of section 2.3.1, one might try to make this transformation local in the form

$$
v^{\mu}(\tau) \longrightarrow e^{\omega_{\alpha \beta}(x)\left(\hat{M}^{\alpha \beta}\right)_{\nu}^{\mu}} v^{\nu}(\tau),
$$

with the LORENTZ generators $\hat{M}^{\alpha \beta}$. Not unexpectedly, this produces an extra term when acted on by $\frac{d}{d \tau}$, namely

$$
\partial_{\rho} \omega_{\alpha \beta}(x)\left(\hat{M}^{\alpha \beta}\right)_{\nu}^{\mu} v^{\rho}(\tau) v^{\nu}(\tau)
$$

where in $\partial_{\rho} \omega_{\alpha \beta}(x)\left(\hat{M}^{\alpha \beta}\right)_{\nu}^{\mu}$ we recognize the index structure familiar from the CHRISTOFFEL symbols in (3). We obtain a modified geodesic equation

$$
\frac{d}{d \tau} v^{\mu}(\tau)+\Gamma_{\nu \rho}^{\mu} v^{\nu}(\tau) v^{\rho}(\tau)=0
$$

with the $S O(3,1)$ connection $\Gamma_{\nu \rho}^{\mu}$ containing in its transformation an inhomogeneous term to cancel (15). One would now have to derive the field strength corresponding to this connection and describe the dynamics of the newly introduced field. This we postpone to the more rigorous treatment in subsequent sections. However, we first take a quick look at an alternative gauge candidate besides the LORENTZ group $S O(1,3)$. 
Instead of rotations one might want to consider gauging the four-dimensional translation group $\mathbb{R}^{(1,3)}$. Similar to (14) the (local) transformation to consider is then

$$
v^{\mu}(\tau) \longrightarrow e^{\epsilon^{\alpha}(x) \hat{P}_{\alpha}} v^{\mu}(\tau),
$$

with the generators $\hat{P}_{\alpha}$ of translations. Although displacements in the tangent fibre cannot be separated from displacements in the base space, we nevertheless distinguish their indices here, and postpone the clarification of the relation between them to the next subsection. The derivative with respect to $\tau$ produces an extra term

$$
v^{\nu}\left(\partial_{\nu} \epsilon^{\alpha}(x)\right) \hat{P}_{\alpha} v^{\mu}
$$

which as above can be cancelled by the inhomogeneous term in the transformation of the $\mathbb{R}^{(1,3)}$ gauge potential $\theta_{\mu}^{\alpha} \hat{P}_{\alpha}$ if one accepts the new geodesic equation

$$
\frac{d}{d \tau} v^{\mu}(\tau)+v^{\nu}(\tau) \theta_{\nu}^{\alpha}(x) \hat{P}_{\alpha} v^{\mu}(\tau)=0 .
$$

Again, the next step would now be to define field strengths and kinetic terms for the gauge Lagrangian. However, we will not proceed any further with this naive approach. Despite all its shortcommings, it should have become clear that when attempting to gauge external symmetry groups one is all too easily confused by the conflation of spatiotemporal and LIE algebra indices. A consistent formulation requires the introduction of orthonormal frames of reference, also called tetrads, which will be one subject of the following subsection. Furthermore, we need to discuss the different possible choices of kinetic terms for the gauge fields of external symmetries, which will turn out to be less restricted than in the YANG-MiLLS case.

\subsubsection{Poincaré Gauge Theory}

Unlike GR, in which the metric plays the fundamental role, gauge-theoretic approaches to gravity including spinorial degrees of freedom require the introduction of tetrads. A tetrad $\theta_{\alpha}^{\mu}(\tau)$ can be pictured as a local frame of reference mediating between the curved (holonomic) spatio-temporal index $\mu$ that coordinatizes the manifold and a set of flat, local (anholonomic) coordinates $\alpha$ such that

$$
g_{\mu \nu}(x)=\eta_{\alpha \beta} \theta_{\mu}^{\alpha}(x) \theta_{\nu}^{\beta}(x),
$$

where $\eta_{\alpha \beta}$ denotes the Minkowski metric.

Clearly, the universality of the gravitational force requires gauging some external symmetry group. But which LIE group should one choose? The equivalence principle states that the curved spacetime of GR remains at least locally Minkowskian. This suggests that the gauge principle should be applied using some rigid symmetry group of Minkowski space. The first attempt to gauge gravity goes back to UtiYAma (1956) (cf. also UtiYAma 1980). While he considered a gauge theory of the Lorentz group $S O(1,3)$, KibBle (1961) and Sciama (1962) proposed to gauge the full PoINCARÉ group ISO $(1,3)$. These programmes have been elaborated, among others, ${ }^{4}$ by HeHL et al. (1976) (cf. also HeHL et al. 1980 and 1995). Our discussion will follow

\footnotetext{
${ }^{4}$ Mention should also be made to the work of IVAnenko and SARdanashvily (1983), Mielke (1987), Ne'EMAN (1980) and Trautman (1980).
} 
mostly these latter accounts. We do not intend to justify the choice of group here. ${ }^{5}$ Recall, however, the discussion in section 2.3.1 of the general framework common to all gauge theories as we know them. It was emphasized there that one always gauges the group corresponding to the conservation of a certain current that will later couple to the interaction field derived in the gauging process. We also know already from GR and its empirical ramifications that gravity certainly (but possibly not exclusively) couples to the energy momentum tensor, the conservation of which is related to spatiotemporal translations. On these grounds it has been argued in favour of an inclusion of the translation part of the POINCARÉ group as (one) ingredient for a gravitational gauge theory intended to generalize GR. It has been claimed for instance that gauging the LORENTZ group only results in seemingly inevitable couplings of LORENTZ gauge fields to the wrong current (cf. HEHL et al. 1980).

Thus attempting to treat the POINCARÉ group following as closely as possible the approach explained in section 2.3.1 we expect four translational and six rotational gauge potentials corresponding to the $(4+6)$ parameters of $I S O(1,3)$. Consider for instance in analogy to (9) a (local) Poincaré transformation acting on a spinor $\Psi(x)$ according to

$$
\Psi(x) \longrightarrow \Psi^{\prime}(x)=e^{\omega^{\alpha \beta}(x) \hat{M}_{\alpha \beta}+\epsilon^{\alpha}(x) \partial_{\alpha}} \Psi(x),
$$

with the six (LORENTZ) rotational generators $\hat{M}_{\alpha \beta}$ and the four generators of translations, namely the partial derivatives $\partial_{\alpha}$ with respect to the anholonomic coordinates. The transformation (21) leads to extra (derivative) terms $\bar{\Psi}(x) i \gamma^{\mu}\left(\partial_{\mu} \omega^{\alpha \beta}(x) \hat{M}_{\alpha \beta}+\partial_{\mu} \epsilon^{\alpha}(x) \partial_{\alpha}\right) \Psi(x)$ in a fermionic Lagrangian of type (8). The first of these can be taken care of by going from the initially partial to a covariant derivative $\partial_{\mu} \longrightarrow D_{\mu}=\partial_{\mu}-\Gamma_{\mu}^{\alpha \beta} \hat{M}_{\alpha \beta}$, with the rotational gauge potential given by $\Gamma_{\mu}^{\alpha \beta}=\partial_{\mu} \omega^{\alpha \beta}(x)$. But the second term cannot be so simply absorbed since it still contains a derivative of the spinor. To solve this apparant problem recall that the Minkowski metric occurs in the CLIFFORD algebra satisfied by the DIRAC matrices. Hence they should carry anholonomic rather than holonomic indices. In Minkowski spacetime there is no need to state this explicitly but the proper starting point for an application of the gauge principle is $\bar{\Psi}\left(i \gamma^{\alpha} \delta_{\alpha}^{\mu} \partial_{\mu}-m\right) \Psi$. We see that the term arising from the local translations can be compensated by adding to the KRONECKER symbol the term $\partial_{\alpha} \epsilon^{\mu}(x){ }^{6}$ The constant $\delta_{\alpha}^{\mu}$ mediating between holonomic and anholonomic indices has turned into the tetrad or translational gauge potential $\theta_{\mu}^{\alpha}$. To summarize, we introduce translational and rotational potentials $\theta_{\mu}^{\alpha}$ and $\Gamma_{\mu}^{\alpha \beta}$. Now what about the field strengths corresponding to these and the Lagrangian determining their dynamics?

\footnotetext{
${ }^{5}$ A certain motivation to consider $I S O(1,3)$ stems from WignER's seminal analysis. The universality of the gravitational interaction (its coupling to all known particles) and the fact that in turn all elementary particles correspond to representations of the POINCARÉ group suggest that this group plays an important role. One could also advocate that supersymmetry and supergravity scenarios are derived from extensions of this group.

${ }^{6}$ We should mention that the precise formulation of this transformation requires to make explicit which system of coordinates the parameters $\epsilon$ refer to, the one before or after the conceptually independent LORENTZ rotation. However, since this is a technicality shedding little light on the issues we are concerned with here, we will not go into details.
} 


\subsubsection{Lagrangians and Field Equations}

Having obtained the gauge potentials we can immediately write down the corresponding field strengths. Similar to (12) we find the translational field strength or torsion

$$
F_{\mu \nu}^{\alpha}=\partial_{\mu} \theta_{\nu}^{\alpha}-\partial_{\nu} \theta_{\mu}^{\alpha}+\Gamma_{\mu \beta}^{\alpha} \theta_{\nu}^{\beta}-\Gamma_{\nu \beta}^{\alpha} \theta_{\mu}^{\beta},
$$

and the rotational field strength, usually referred to as curvature,

$$
F_{\mu \nu \alpha}^{\beta}=\partial_{\mu} \Gamma_{\nu \alpha}^{\beta}-\partial_{\nu} \Gamma_{\mu \alpha}^{\beta}+\Gamma_{\mu \gamma}^{\beta} \Gamma_{\nu \alpha}^{\gamma}-\Gamma_{\nu \gamma}^{\beta} \Gamma_{\mu \alpha}^{\gamma}
$$

Such a geometry with both non-vanishing curvature and torsion is called a RIEMANN-CARTAN space-time $\mathbb{U}_{4}$. Setting the torsion equal to zero one obtains a (pseudo-)RIEMANNian space-time $\mathbb{V}_{4}$, whereas for vanishing curvature the space-time is a teleparallel $\mathbb{T}_{4}$.

There are some important points to mention here. Whereas for global PoINCARÉ transformations all the ten generators were required this is not strictly so for the local action of the group, at least not from the point of view of pure mathematics. Since the group of local translations is nothing but the diffeomorphism group (compare section 2.3.3), it already implicitly contains also the rotations. However, from an applied mathematical or physical point of view there exists a crucial difference. The practising physicist distinguishes the freedom of coordinatizing the base manifold from that of changing to a different kinematic system of reference. In other words, a particular choice of spatiotemporal coordinate system still allows one to perform a conceptually independent LORENTz boost.

Caution should be excercised not to confuse the connection $\Gamma$ introduced in the previous paragraphs with the LEVI-CIVITA connection \{\} featuring in GR as formulated on $\mathbb{V}_{4}$. Before we can express the relation between the two first note that contraction with a tetrad enables one to transform from holonomic to anholonomic indices and vice versa. One can then show that

$$
\Gamma_{\mu \nu}^{\lambda}=\{\}_{\mu \nu}^{\lambda}-K_{\mu \nu}^{\lambda}
$$

with Christoffel symbols \{\}$_{\mu \nu}^{\lambda}$ and the contorsion tensor $K_{\mu \nu}{ }^{\lambda}=\frac{1}{2}\left(-F_{\mu \nu}{ }^{\lambda}+F_{\nu}{ }^{\lambda}{ }_{\mu}-F_{\mu \nu}^{\lambda}\right)$.

One should not yet jump to conclusions concerning the gauge character of usual GR. It might seem that since torsion is absent in EINSTEIN's theory only the LORENTZ group deserves attention. One is not really in a position to decide on this yet since these questions require investigating the different Lagrangians allowed in the $\mathbb{U}_{4}$ framework.

Recall that in ordinary gauge theories there was not really much freedom in choosing the Lagrangian (13) for the gauge fields. Not only was their coupling to the fermionic fields dictated by a correct application of the gauge principle, but also their kinetic term constrained to $F_{\mu \nu}^{a} F^{a \mu \nu}$ by the requirements of invariance under gauge and coordinate transformations and the limitation to second order field equations. In the gravitational case under consideration here one has more freedom since holonomic and anholonomic indices can be interchanged by multiplying with a tetrad. Contract for example the translational field strength $(22)$ with a tetrad $\theta_{\alpha}^{\lambda}$ to find $F_{\mu \nu \lambda}$. This opens up new options for the kinetic term $\sim F^{2}$ in the gauge Langrangian. To be precise, keeping in mind the antisymmetry in $\mu$ and $\nu$, one now has $(3 \times 2) / 2=3$ possibilities. The curvature (23) can be treated in a similar manner. In contrast to this, there was no way to turn an internal $a$ into an external $\mu$ index in the YANG-Mills case. Schematically, the four allowed 
terms in the PoINCARE gauge Lagrangian are given by ${ }^{7}$

$$
\mathcal{L} \sim \text { const. }+(\text { curvature scalar })+(\text { torsion })^{2}+(\text { curvature })^{2} .
$$

Note the novel feature of a curvature scalar which can be seen as a direct consequence of the mixing mechanism of curved and flat indices mentioned above.

Choosing only the first two possible terms for the Lagrangians, namely the constant and the curvature scalar, one obtains the EInSTEIN-CARTAN-SCIAMA-KIBBLE (ECSK) theory. It contains corrections to GR quadratic in the spin but for spinless matter reduces to EINSTEIN's original theory. In GR one can thus consider the unique LEVI-CIVITA connection as resulting from the particular choice of Lagrangian. ${ }^{8}$

But the ECSK theory is not the only possible choice of Lagrangian leading in the limit of spinless matter to the same predictions as GR. A pure translational gauge field theory with a particular choice of quadratic Lagrangian (recall the extra freedom discussed above in juxtaposition to the YANG-Mills case) fulfills this criterion equally well. ${ }^{9}$ Historically, this was first done by Cho (1976) (cf., however, also Feynman et al. 1995). He considers a scalar field instead of the Dirac Langrangian we gauged in (21), and then derives the specific translational gauge Lagrangian equivalent to GR through a LORENTZ transformation consistency condition on the scalar field. ${ }^{10}$ This approach does not at all necessitate the introduction of fermionic fields and thus provides a theory to be justifiably considered empirically equivalent to GR, but at the same time conceptionally clearly distinct from it.

How the seemingly different geometric but nevertheless empirically equivalent approaches of these two theories can be reconciled is most clearly demonstrated with the help of equation (24). For the case of vanishing rotational field strenth also $\Gamma_{\mu \nu}^{\rho}$ can be transformed to zero in a particular system of coordinates. We then obtain the equality ${ }^{11}$

$$
\{\}_{\mu \nu}^{\lambda}=K_{\mu \nu}^{\lambda}
$$

\footnotetext{
${ }^{7}$ More explicitly, the Lagrangian can be written (cf. HeHL 1981, p. 121; this German paper provides the most comprehensive formula, but the general line is of course also contained in the other HEHL papers mentioned above)

$$
\begin{aligned}
\mathcal{L} \sim & \frac{1}{(\mu l)^{4}}+\frac{1}{l^{2}}\left[\frac{1}{2 \chi} F_{\alpha \beta}^{\beta \alpha}+\frac{1}{4} F_{\alpha \beta}^{\gamma}\left(d_{1} F_{\gamma}^{\alpha \beta}+d_{2} F_{\gamma}^{\beta \alpha}+d_{3} \delta_{\gamma}^{\beta} F_{\delta}^{\alpha \delta}\right)\right] \\
& +\frac{1}{4 \kappa} F_{\beta \alpha \gamma \delta}\left(F^{\alpha \beta \gamma \delta}+f_{1} F^{\alpha \gamma \beta \delta}+f_{2} F^{\gamma \delta \alpha \beta}+f_{3} \eta^{\alpha \delta} F_{\epsilon}{ }^{\beta \gamma \epsilon}+f_{4} \eta^{\alpha \delta} F_{\epsilon}{ }^{\gamma \beta \epsilon}+f_{5} \eta^{\alpha \delta} \eta^{\beta \gamma} F_{\epsilon \kappa}^{\epsilon \kappa}\right) .
\end{aligned}
$$

Here $l$ denotes the Planck length, $\mu$ is related to the cosmological constant, $\chi$ measures the relative strenths of the squares of torsion and curvature respectively, and $\kappa$ is a kind of coupling constant for the LoRENTz potential. As already mentioned there is more freedom here than in the usual gauge theories also in the terms quadratic in the field strengths. They are not uniquely determined but contain the free parameters $d_{1}$ to $d_{3}$ and $f_{1}$ to $f_{5}$ (however, of the latter only four are independent).

${ }^{8}$ In the variational approach to ordinary GR such a feature can be expressed in the PALATINI approach, where the symmetry constraint of the CHRISTOFFEL symbols are obtained as a second field equation, varying the Lagrangian as it were with respect to a physically non-propagating field.

${ }^{9}$ Taking only terms quadratic in the torsion and setting $d_{1}=-\frac{1}{2}, d_{2}=-1$ and $d_{3}=2$ in (25) one obtains a gauge field theory of the translational group equivalent to GR to all orders. This version is called theory $T_{4}$ in this paper.

${ }^{10}$ The idea is to rewrite (6) in terms of the tetrads so that the quadratic structure in the field strength becomes apparent, namely $\mathcal{L}_{G R} \sim F^{2}$ (up to a divergence) with torion $F_{\mu \nu}^{\alpha}=\partial_{\mu} \theta_{\nu}^{\alpha}-\partial_{\nu} \theta_{\mu}^{\alpha}$ from (22) and $\Gamma=0$.

${ }^{11}$ Note that this equation only holds in a particular coordinate system or gauge, as after all $K_{\mu \nu}^{\lambda}$ is a tensor whereas \{\}$_{\mu \nu}^{\lambda}$ transforms inhomogeneously.
} 
expressing the possibility of describing the geometries underlying the two physical theories either in terms of the familiar LEVI-CIVITA connection or making use of the contorsion tensor. It will be the subject of section 3 to discuss in detail this apparant indeterminacy of geometry.

\subsection{Curve It, Gauge It, or Leave It?}

After briefly reminding the reader of the geometrical structures of GR and introducing a LORENTZ-invariant gravitational theory in flat spacetime (flat GR), we have described in this section the concepts of gravitational gauge theory. One starts from a fundamental spinor whose external symmetry, in our case the PoIncARÉ group, is then gauged. In this sense they are similiar to standard YANG-MILls theories, but crucial differences remain. In the latter case the fibres constitute internal symmetry spaces which originate from quantum symmetries, whereas for the former external space-time is gauged. As a consequence these gauge attemps naturally incorporate the concept of universality so particular to the gravitational interaction. No field (carrying energy) can escape gravity since, in fibre bundle parlance, its gravitational effects are not limited to internal spaces. This feature is sometimes referred to as the soldering of base space and fibres. Also, the choice of Lagrangian turns out to be less constrained for gravitational theories than for their YANG-MiLls counterparts, where it is always quadratic in the field strength.

Two of the gravitational gauge theories discussed, the LORENTz gauge theory $T_{3}$, and also the pure torsion theory $T_{4}$, in the limit of point matter both become equal to GR in all their empirical predictions, albeit derived from a conceptually rather different approach, resulting even in a different discription of the underlying geometry. ${ }^{12}$ This is related to the fact that in the case of POINCARÉ gauge theory we may have different types of bundle curvature, namely Riemann curvature as well as torsion. Our choice to either curve $\left(T_{1}\right)$, gauge $\left(T_{3}, T_{4}\right)$ or just leave the geometry of spacetime unaltered $\left(T_{2}\right)$ seems conventional or indetermined.

\section{Indeterminacies in Gravitational Theories}

"Three theses of indeterminacy have figured conspicuously in my writings: indeterminacy of translation, inscrutability of reference and underdetermination of scientific theory."

QUine (1990)

In the terminology of QUINE the main emphasis of this work lies on indeterminacy in the sense of underdetermination of scientific theory, however, in this section we shall apply both the issue of inscrutability of reference and theory underdetermination to the four gravitational theories $T_{1}$ up to $T_{4}$ considered in the preceding section (indeterminacy of translation is of no importance to us). We start with the underdetermination issue.

\footnotetext{
${ }^{12}$ One might want to critize our analysis for the detour taken via spinorial fields before finally returning to the limit of scalar matter. Albeit not so easily refuted for $T_{3}$, this argument can be repudiated for $T_{4}$, for which a treatment without recourse to spinors does exist.
} 


\subsection{Theories, Models, and the Issue of Underdetermination}

As already mentioned in the introductory section 1, theory underdetermination by empirical evidence rests on the supposition that the set of theory terms necessarily exceedes that of observation terms, so as to render impossible any direct correspondence between the theoretical and observational parts of the theory. The debate about theory underdetermination has centred around a number of focal points which deserve careful separation: Firstly, the distinction between theories and mere models of theories. Secondly, the question has been asked how many competing theories there are: infinitely many - maybe even automatically generated - as opposed to a final number of rivals - maybe even just two. Thirdly, the juxtaposition final versus non-final theories arises and, finally, for all of these cases the apparent question of good examples. This latter question has been a strong stimulation for this work, since we believe that theories $T_{1}$ to $T_{4}$ do indeed represent good examples for a case study of theory underdetermination - at least in the sense of what in the final section 4 we propose to call practical underdetermination.

We discuss first the distinction between theories and models. A model can be viewed as an interpretation of a given mathematical structure, assigning certain truth conditions to its sentences. In this way a hitherto merely formal structure is endowed with meaning and semantics. Models are predetermined up to isomorphisms - as opposed to theories, which are considered classes of isomorphic models. ${ }^{13}$ As an example, the reader might recall REICHENBACH's idea of universal forces, which act uniformly on all materials irrespective of their composition. Clearly, gravitation provides such a force. Given a gravitational configuration of all matter in space we might as well presume another configuration which differs from the former by a certain universal - and therefore unobservable - deformation of our measuring rods. This possibility of a universal force establishes the idea of conventionalism in spacetime theories.

This is nicely illustrated by the so-called "hollow earth theory", which goes back to the American Cyrus TeED of the late 19th century, but later on also became a real cult in Germany (cf. GARDNER 1952, chap. 2). Instead of propagating the intuitive picture of a convex earth, proponents of this "theory" claim that we are living on the inside of a concave world with formerly straight lines turned into circles through the centre. Of course, the simple polar coordinate mapping $r^{\prime}=\frac{R^{2}}{r}$ ( $R$ radius of the earth) allows to shift back and forth between the two picturesunmasking them as diffeomorphic models of one and the same theory. ${ }^{14}$ The hollow earth theory thus constitutes a viable, non-falsifiable alternative to the usual convex point of view. The same holds for models of spacetime theories including universal forces.

However, such interpretational issues, as necessarily implied in our discussion about theories and models, always depend crucially on ontological commitments. To a spacetime substantivalist, for instance, two diffeomorphic models of one and the same spacetime represent different physical situations: the state of the world has changed in going from one to the other-despite its unobservability. Consequently, any strict distinction between theories and models must fail due to its sensitive dependence on ontological committments and their indispensible, possibly

\footnotetext{
${ }^{13}$ We do not subscribe to a specific notion of scientific theories, as for instance statement vs. non-statement view. Clearly, our definition of a model just given displays close affinity to the modern semantic viewpoint. For our general argument, however, neither opinion has to be preferred.

14 As SeXl (1983) has argued, the $r=0$ singularity can be cured by making suitable symmetry assumptions (cf. section 4).
} 
implicit, criteria of empiricity. For the sake of our analysis, however, we shall not spend too much time on far-reaching ontological considerations. We will rather subscribe to the practising physicist's point of view: Modern gravitational theories always consist of classes of (infinitely many) diffeomorphic models, simply because they utilize differentiable manifolds to represent spacetime. For the investigation of theory underdetermination it will therefore not be sufficient to consider only different models, since these merely constitute isomorphic images of one and the same theory. By way of contrast, distinct theories differ by semantic changes, i.e. differences in their theoretical terms and entities predicted. As we will argue in the following, our four examples $T_{1}$ to $T_{4}$ do provide such semantical changes - while simultaneously leaving the observational part unaltered - and are therefore suited for a case study of theory underdetermination.

\subsection{Ontological Difference-Topological Indifference}

Whereas for $T_{1}$ (standard GR) the gravitational field "lives" on a RIEMANNian manifold, i.e. curved spacetime, in $T_{2}$ (flat GR) the manifold is substituted by its flat approximation, i.e. Minkowski spacetime. This apparent logical — and ontological - difference justifies talking about two different theories. Although these share to a large extent the same theoretical entities, the most fundamental one, namely the metric tensor $g_{\mu \nu}$ of $T_{1}$, has been replaced in $T_{2}$ by $h_{\mu \nu}$ together with a fixed Minkowski background spacetime.

Clearly, equation (7) expresses the indistinguishability between $T_{1}$ and $T_{2}$ by any local experiment. But what about global aspects, i.e. cosmic models? A globally closed spacetime - such as the EINSTEIn cosmos ${ }^{15}$ c could hardly be a solution of $T_{2}$ 's field equations in flat space. We may approximate this model to the best we can, but one point of closure still remains missing. Thus, in applying GR to spacetime in its totality there seems to arise - at least in principle - an empirical difference between $T_{1}$ and $T_{2}$.

There are two objections at hand, pertaining to the foundations of GR: Firstly, it is not clear whether we may apply the theory to the universe in toto (that is, GR should perhaps rather be considered a local field theory). Secondly - the more intriguing objection-global topology is neither in GR nor in any other gravitational theory part of the dynamics, but rather fixed by the initial conditions. More explicitly, suppose we were living in a closed universe. We would then ascribe this closure property to an additional physical effect. Now let $E$ be an explanation of this effect, then $T_{2}+E$ will have the same predictive power as $T_{1}+E{ }^{16}$ Modern gravitational theories prove to be topologically indifferent and, hence, $T_{1}$ and $T_{2}$ share the feature of topological indifference, while they are logically different. ${ }^{17}$

\footnotetext{
${ }^{15}$ This reasoning applies to any solution with non-trivial global topology, such as the FrIEDMANN-ROBERTSONWALKER metrics.

${ }^{16}$ One could stipulate some $E$ compatible with only one of these two theories. Without $E$, however, on the basis of currently available data no final judgement on this point can be made.

${ }^{17}$ To be sure, the problem of topology change in GR is longstanding and non-trivial. It has been argued that changes in topology are in principle possible, and thus our statement about topological indifference seems too naive. However, topology changes come at a high price. They require the relaxation of energy conditions or time orientability, i.e. the existence of closed timelike curves (basically due to theorems of GEROCH and TIPLER; for a recent discussion see CALLENDER and WEINGARD 2000). If one is not willing to pay such prices, our argument in this paragraph still holds. Otherwise, the possibility of topological changes in principle applies to any of our theories $T_{1}$ to $T_{4}$ equally well, allowing closed models even within approaches $T_{2}$ to $T_{4}$. Similar considerations
} 
Let us now turn to theories $T_{3}$ and $T_{4}$. In gauge theories of gravity we observe a considerable enlargement of the geometrical arena, provided by the total space of principal bundles as well as their associated vector bundles. In $T_{3}$ and $T_{4}$ a differentiable manifold (with LORENTZ signature) is used as spacetime base space and a representation of either the LORENTZ group or the translation group as fibre spaces of the principal bundle. For both of them the vector bundle is isomorphic to the tangent bundle of the manifold. A natural way to gauge theories of gravity is the route from $T_{1}$ to $T_{3}$ : take standard GR and make use of its already built-in bundle structure in terms of the LEVI-CIVITA connection as a one-form taking values in the LIE algebra of the homogeneous LORENTz group. Clearly, the collection of observation sentences remains the same. However, in this process the status of the fundamental entities has changed. The metrical tensor $g_{\mu \nu}$ no longer plays a primary role, but tetrads and connections as well as the curvature tensor (now considered as generalized curvature in bundle space) have taken over.

\subsection{Inscrutability of Reference: The Habitat of the Gravitational Field}

The foregoing discussion leads us straight into the problem of reference in ontology and its alleged inscrutability according to (QUINE 1960). Unlike in YANG-MILLs theories, in gauge theories of gravitation the local product of base space and fibre is not just an artificial one involving two independent and genuinely distinct spaces, but there exists a soldering of them (cf. section 2.4). Fibres and base space are inextricably intertwined, which can most prominently be seen from the isomorphism between the vector and tangent bundles. Again, we could ask where the gravitational gauge fields - tetrads, connections, curvature - "live", and as a consequence of the soldering find the reference relation between gauge theoretic entities and bundle geometry indetermined. We simply cannot say whether the fields live in the fibres or in base space. Thus, the soldering - as a peculiarity of fibre bundle theories of gravity - provides us with an intriguing example of the inscrutability of reference (also ontological relativity according to QUINE).

Note, however, that this ontological indeterminacy must prima facie be distinguished from theory underdetermination. In contrast to the latter, inscrutability of reference just applies to different models of one and the same theory. Clearly, theories $T_{3}$ and $T_{4}$ differ in their semantics from $T_{1}$ and $T_{2}$, and are therefore examples of theory underdetermination, but this leaves the ontologically indetermined question of where the fields live unaffected. Therefore, a proponent of $T_{3}$ or $T_{4}$ may or may not be a bundle space substantivalist, ${ }^{18}$ the theories themselves do not favour either point of view.

\subsection{Inscrutability of Reference: Curvature versus Torsion}

As explained in section 2.3.3, the homogeneous LORENTZ group is not the only possible gauge group to mimic GR - the group of POINCARÉ translations can serve the same purpose. A translational gauge theory fits even more nicely into the gauge theoretic framework: Firstly, it includes the correct coupling to the desired NoETHER current. Secondly, local translations are isomorphic to diffeomorphisms and, hence, $T_{4}$ may equally well be considered a gauge theory

apply to the conventionality of topology, resulting from some reidentification procedure of points (as has been discussed in Glymour 1973).

${ }^{18}$ Cf. LyRe (1999). 
of the diffeomorphism group (these groups provide isomorphic models of $T_{4}$ ). ${ }^{19}$ Thirdly, the Lagrangian is quadratic in the field strength and therefore displays a close analogy to ordinary YANG-MILls theories.

Most remarkably, $T_{4}$ constitutes a pure torsion theory unlike the curvature approaches $T_{1}$, $T_{2}$ and $T_{3}$. It thus clearly differs semantically from its three rivals. However, due to equality (27), $T_{3}$ and $T_{4}$ are mathematically equivalent. One might very well wonder whether we could in fact be considering just two different models of one and the same theory. We are required to state more precisely which "semantical differences" suffice for a distinction of theories. The following criterion seems reasonable: For distinct theories $T$ and $T^{\prime}$ their semantics should not be translatable into each other, i.e. theoretical terms of $T$ cannot be transformed into theoretical terms of $T^{\prime}$ (or reformulated within the context of $T^{\prime}$ ), nor vice versa. Once, however, there exists a dictionary between the two theory contexts, they are clearly but two equivalent formulations. A trivial example of just switching the terms "electron" and "molecule" in physics is due to Quine (1975) (alternatively "electron" and "proton" in Quine 1990). This is exactly what happens also in the case of the hollow earth theory: The polar coordinate mapping between convex and concave points of view provides a dictionary between two semantical models of one and the same theory. Now, doesn't (27) provide such a dictionary for the case considered here?

A first answer could be in the affirmative. The choice between curvature and torsion seems just a matter of convention. However, there is a subtle catch: PoINCARÉ gauge theory (PGT) provides a unification of theories $T_{3}$ and $T_{4}$, as illustrated in section 2.3.3. It is - for many meta-theoretic reasons outlined in 2.3.3 (such as the analogy to YANG-MiLLS and the inclusion of spinning matter) - a very reasonable candidate for a more advanced gauge theory of gravity, which includes both curvature and torsion as two distinct features. This possibility of unification at a higher theoretical level does neither exist for the case of the hollow earth theory nor for any other, even more trivial example of mere translations of one and the same theory. ${ }^{20}$

We shall end this section with a further remark. Topological indifference, as first introduced for $T_{2}$, also applies to theories $T_{3}$ and $T_{4}$. However, here the problem is even more severe. In $T_{2}$ we are certainly working with flat Minkowski space. What, then, is the nature of base space in gauge theories of gravity? Clearly, the gauge principle starts from flat space (see

\footnotetext{
${ }^{19}$ WeInStEIn (1999) seems to overlook this isomorphism, since he has argued that “... the diffeomorphism group is ... not a gauge group in the specific sense this term has in particle physics". He then falsely concludes that "... the diffeomorphism group is not the automorphism group of the principle fiber bundle". However, as for instance TRAUTMAN (1980) concisely points out: “... in the theory of gravitation, the group $\mathcal{G}_{0}$ of "pure gauge' transformations reduces to the identity; all elements of $\mathcal{G}$ correspond to diffeomorphisms". Here, $\mathcal{G}$ denotes the group of local gauge transformations, which is quite generally a subgroup of the automorphism group of the principle bundle. Hence, in gauge theories of gravity the automorphism group of the principle bundle is $a$ fortiori isomorphic to the diffeomorphism group, which, again, expresses nothing but the soldering and, thus, the inscrutability of reference of the habitat of the gravitational field.

${ }^{20}$ Yet another feature of $T_{4}$ undermines regarding it as identical to $T_{3}$. We have argued that - due to the bundle soldering - gauge theories of gravity are ontologically indetermined as to the question where the fields live. However, the translational gauge theory poses a certain problem for the straightforward bundle theoretic point of view. Local translations can live in the fibres as well as in base space, where they represent infinitesimal shifts. In bundle space it is therefore impossible to stay in the same fibre (above a fixed base point). This subtlety requires the introduction of richer mathematical structures - such as affine bundles and the breaking of translational gauge symmetry - which do not feature in $T_{3}$ (cf. GRONwALd 1998). In our opinion, these "subtleties" provide further support for considering $T_{3}$ and $T_{4}$ as distinct theories.
} 
section 2.4). Topological indifference in a sense prevents a meaningful distinction between flat and curved base spaces. As we have seen already, the same holds for gauge theories of gravity due the soldering property. Thus, topological indifference as well as ontological indeterminacy intrinsically overlap, undermining a fortiori the decision of whether we inhabit a flat or a curved world.

\section{Practical Underdetermination of Non-final Theories}

We have argued that theories $T_{1}$ to $T_{4}$ provide good examples for a case study of theory underdetermination by empirical evidence. This statement holds at least from the practising physicist's point of view - and this chapter is intended to elaborate on exactly this proviso. Surely, $T_{1}$ to $T_{4}$ meet the criterion of empirical equivalence. On the other hand, their theoretical contexts are incompatible, i.e. theoretical terms of one theory cannot be reformulated within the context of the other. Hence $T_{1}$ to $T_{4}$ differ, in practice, in their theoretical entities assumed. We are justified to consider them as more than mere models of one and the same theory.

We have to admit, again, that our distinction between theories and models is not clear-cut. We have mentioned QuiNE's switching of "electron" and "proton", and have compared this with the hollow earth example, where a simple dictionary between convex and concave points of view is given by: straight line $=$ circle , infinitely distant point $=$ centre point , centre of earth $=$ infinitely distant point. PoINCARÉ's conventionality of empirical geometry provides a similar example. An infinite space measured by rigid (i.e. length invariant) rods can easily be mapped to a finite space measured with rods uniformly shrinking as they move away from the centre. We simply have to stipulate the choice of our length measuring device by convention as either rigid under transport or varying in a determined way. However, both examples point to a difficulty in assuming theoretical terms in one picture which do not occur in the other: the centre of the convex earth as well that of POINCARÉ's finite space. In order to reformulate the two "centres" one has to introduce additional assumptions (cf. footnote 14). Now, doesn't this look more like different theories instead of mere models? Remarkably, Poincaré's conventionalism example was the

only non-trivial one QuINE has ever mentioned in support of his underdetermination thesis (to the best of our knowledge). And, even more remarkably, he himself seems to have changed his mind on its status over time. Whereas in his classic 1975 paper he considers Poincaré's two pictures as formulations of a single theory, he points out in (1990) their inconvertability resulting from conflicting theoretical terms. He then continues to speak of "drastically unlike theory formulations".

We would like to stress that all these examples, indeed, lie somwhere on a continuum with the doctrines of conventionalism and underdetermination as its two extremes. Whether we call them "drastically unlike" becomes a matter of ontological commitment as well as meta-theoretical criteria - to the practising physicist a matter of intuition. From this point of view, however, neither the hollow earth picture nor POINCARÉ's example cause real worries for scientists today. To them, they certainly constitute merely reformulations of a single theory. We believe, however, that this is not so for the case of our examples $T_{1}$ to $T_{4}$, which in fact differ considerably also from the modern practising physicist's point of view.

In order to expand on this point of view in relation to underdetermination we now return to the questions of how many and final versus non-final theories, and afterwards to the issue of 
good examples as raised at the beginning of section 3. In the underdetermination debate little attention has been paid - neither by QUINE nor others - to concrete examples, mainly for the following three reasons: Firstly, on fundamental grounds the thesis has to apply to any theory and not just to a few examples. Secondly, the thesis should apply to - in principle -infinitely many rivals (which - in principle - could be automatically generated ${ }^{21}$ as a consequence of the first reason). Thirdly, it should apply only to final theories - cf. HoEfer and RosenberG (1994), or, as QUINE (1975) puts it: "If all observable events can be accounted for in one comprehensive scientific theory-one system of the world ...-then we may expect that they can all be accounted for equally in another, conflicting system of the world. We may expect this because of how scientists work. For they do not resist with mere inductive generalizations of their observations: mere extrapolations to observable events from similar observed events. Scientists invent hypotheses that talk of things beyond the reach of observation. The hypotheses are related to observation only by a kind of one-way implication; namely, the events we observe are what a belief in the hypotheses would have led us to expect. These observable consequences of the hypotheses do not, conversely, imply the hypotheses. Surely there are alternative hypothetical substructures that would surface in the same observable ways. Such is the doctrine that natural science is empirically under-determined ... by all observable events."

We very much agree with QUINE in the general way he presents his argument - with particular emphasis, as the reader may guess already, on the pragmatic core character of his thesis ( "... of how scientists work"). From such a pragmatic point of view there appears little need to accept the thesis as principally valid. ${ }^{22}$ Should we, thus, really exclude the possibility of good practical examples for a finite number of non-final theories?

Some concrete examples of theory underdetermination can indeed be found in the literature. The most common ones taken from physics are again due to the conventionalism of geometry in spacetime theories. A second example often advocated is the debate between Copenhagen and Bohmian quantum mechanics. This, indeed, might be a good example, but one is immediately faced with the standard objection against non-final theories: the provisional status of science today. We simply do not (yet) have a final theory of everything at our disposal for interpretation. ${ }^{23}$ Some day we might perhaps very well be able to decide between Copenhagen and Bонм - either by means of a new theory which simply replaces its predecessor(s), or, more probably, through certain meta-criteria (for instance, the notorious problem of incorporating LORENTz invariance into BoHM's theory). By way of contrast, a final theory will already entail any observation conditionals needed to judge the status of its possibly equivalent rivals.

In order to avoid for the time being the difficulties connected with incomplete knowledge in non-final theories, one might consider examples of "earlier" systems of the world, e.g. NewTONian mechanics with or without absolute space (VAN FrAASSEN 1980). In fact, in NEWTONian

\footnotetext{
${ }^{21}$ See KUKLA (1993) for a proposal.

${ }^{22}$ For further conceptual doubts concerning the underdetermination thesis see LAUDAN and LEPLIN (1991) and LEPLIN and LAUDAN (1993).

${ }^{23}$ In fact, the idea of a final theory, as we use it here, may be understood in two ways. QuiNE's system of the world is primarily thought of as a simple conjunction of all observation sentences covering all observable events. Physicists, however, think of it as a theory of everything, a theoretical framework which in principle allows to deduce all known interactions. As opposed to a mere conjunction of observation sentences, such a programme is characterized by a striving for unification and generalization.
} 
times there was no way to distinguish between the two theories, which is why they indeed justify talking about theory underdetermination - at that time. However, as we know today, NewTON's concept of absolute space was fatally flawed. Overcoming it was one of the crucial cornerstones in EINSTEIn's discovery of special relativity. Hence there sometimes do exist practical examples of theory underdetermination for non-final theories which most certainly are of just provisional status. These are cases of, as we propose to call them, practical underdetermination of non-final, rivaling theories. Not strict examples of theory underdetermination, they nevertheless - and this seems to us the crucial point - provide very intriguing fields of study, since they point to incomplete scientific knowledge! Practising scientists pay attention to them, since they may be fruitful for questions of scientific progress, especially in observation-starved fields. Concrete practical examples of theory underdetermination can be seen as possible guidelines for the question of where and how scientific efforts should focus.

From this point of view let us re-iterate theories $T_{1}$ to $T_{4}$. It is of course highly plausible to assume the provisional status of todays's theories of gravity, but we propose to not stigmatize this non-finality for philosophical reasons. What, then, are the crucial meta-theoretic criteria to decide between the existing rivals? In section 2.3 we have already mentioned some: gauge theories of gravity allow for more generalized Lagrangians, the inclusion of spinning matter as well as the possibility of YANG-MiLls-like approaches. Scientists welcome at least some of these features for their unifying potential, which has led LIU (2001) to investigate carefully the pros and cons of already accomplished as well as proposed unification programmes. We are at this point less interested in giving a formal, possibly dogmatic list of criteria to be met by a scientific description in order to deserve being called unifying. Rather, we would like our line of arguments to be read as a plea against misunderstood conservativism in science. For the case of gravitational theories, standard GR is often praised for its alleged beauty but little notice is taken of alternative accounts.

Strict theory underdetermination results in the absence of real examples - at least for the case of non-final theories. On the other hand there do exist good practical examples, namely theories $T_{1}$ to $T_{4}$. We also believe that the Copenhagen vs. BoHm debate is of more or less the same quality. It indicates that the interpretational debate about quantum theory touches open questions, possibly leading to an eventually deeper understanding of the nature of the quantum. Note the non-triviality of this statement: We provide an argument against a merely positivistic point of view towards quantum mechanics. Yet another good example may arise from the analysis of topological aspects of gauge theories, such as the AHARONOV-BoHm effect (EYNCK et al. 2001).

The abstract thesis of theory underdetermination may or may not pose a problem for scientific realism, as sometimes stated, the practical underdetermination of non-final theories for sure does not have such aftermaths. Its consequences are much more practical and indeed productive: good examples of practical underdetermination point to open questions in our understanding of nature even in the absense of disagreements between theory and observation, and thereby promote scientific progress. 


\section{Acknowledgements}

We would like to thank F. W. HeHL and his group at the University of Cologne as well as F. GRonwald for clarifying discussions on technical issues of gauge theories of gravitation. We also acknowledge helpful remarks by N. VON RUMMELL on the philosophical parts of the paper.

\section{References}

Auyang, S. Y. (1995). How is Quantum Field Theory Possible? Oxford University Press, New York.

Barbour, J. B. (1967). Zur Herleitung der Einsteinschen Gravitationstheorie aus den Prinzipien klassischer lorentzinvarianter Feldtheorien. PhD thesis, Universität zu Köln.

Callender, C. and N. Huggett, eds. (2001). Physics meets Philosophy at the Planck Scale. Cambridge University Press, Cambridge.

Callender, C. and R. Weingard (2000). Topology Change and the Unity of Space. Studies in History and Philosophy of Modern Physics, 31:227-246.

Cho, Y. M. (1976). Einstein Lagrangian as the translational Yang-Mills Lagrangian. Physical Review D, 14(10):2521-2525.

EArman, J. (2001). Gauge Matters. (PSA 2000 conference lecture, E-print PITT-PHILSCI00000070).

Eynck, T. O., H. Lyre and N. v. Rummell (2001). A versus B! Topological nonseparability and the Aharonov-Bohm effect. Conference lecture at "Quantum Structures V", Italy. (Eprint PITT-PHIL-SCI00000404).

Feynman, R. P., F. B. Morinigo and W. G. Wagner (1995). Feynman Lectures on Gravitation. California Institute of Technology.

Gardner, M. (1952). Fads and Fallacies in the Name of Science. Dover, New York. (Second edition 1957).

Glymour, C. (1973). Topology, Cosmology and Convention. In Suppes, P., ed. Space, Time and Geometry. Reidel, Dordrecht.

Gronwald, F. (1998). A Note on Gauge Covariant Translations in the Gauge Approach to Gravity. Acta Physica Polonica B, 29(4):1121-1129.

Gupta, S. N. (1952). Quantization of Einstein's Gravitational Field: Linear Approximation. Proceedings of the Royal Society of London, 65A:161-169.

Gupta, S. N. (1957). Einstein's and Other Theories of Gravitation. Reviews of Modern Physics, $29: 337$. 
Hehl, F. W. (1981). Zur Eichfeldtheorie der Gravitation. In Nitsch, J., J. Pfare and E.W. Stachow, eds. Grundlagenprobleme der modernen Physik. B.I.-Wissenschaftsverlag, Mannheim.

Hehl, F. W., P. v. D. Heyde, G. D. Kerlick and J. M. Nester (1976). General Relativity with Spin and Torsion: Foundations and Prospects. Reviews of Modern Physics, 48:393-416.

Hehl, F. W., J. D. McCrea, E. W. Mielke and Y. Ne'eman (1995). Metric-Affine Gauge Theory of Gravity: Field Equations, Noether Identities, World Spinors, and Breaking of Dilation Invariance. Physics Reports, 258:1-171.

Hehl, F. W., J. Nitsch and P. V. D. Heyde (1980). Gravitation and the Poincaré Gauge Field Theory with Quadratic Lagrangian. In HELD (1980).

Held, A., ed. (1980). General Relativity and Gravitation: One Hundred Years after the Birth of Albert Einstein. Plenum Press, New York. 2 Volumes.

Hoefer, C. and A. Rosenberg (1994). Empirical Equivalence, Underdetermination, and Systems of the World. Philosophy of Science, 61:592-607.

Ivanenko, D. and G. Sardanashvily (1983). The Gauge Treatment of Gravity. Physics Reports, 94(1):1-45.

Kibble, T. W. B. (1961). Lorentz Invariance and the Gravitational Field. Journal of Mathematical Physics, 2:212.

Kukla, A. (1993). Laudan, Leplin, Empirical Equivalence and Underdetermination. Analysis, $53: 1-7$.

LAUdAn, L. and J. LePLin (1991). Empirical Equivalence and Underdetermination. Journal of Philosophy, 88:449-472.

Leplin, J. and L. LAudAn (1993). Determination Underdeterred: Reply to Kukla. Analysis, $53: 8-16$.

LIU, C. (2001). Gauge Gravity and the Unification of Natural Forces. (E-print PITT-PHILSCI00000364).

Lyre, H. (1999). Gauges, Holes, and their 'Connections'. Conference lecture at "History and Foundations of General Relativity 5", Notre Dame, Indiana. (E-print gr-qc/9904036).

Lyre, H. (2000). A Generalized Equivalence Principle. International Journal of Modern Physics D, $9(6): 633-647$.

Lyre, H. (2001). The Principles of Gauging. Philosophy of Science, 68(Proceedings):S371-S381.

Mielke, E. W. (1987). Geometrodynamics of Gauge Fields. Akademie-Verlag, Berlin.

Mittelstaedt, P. and J. B. Barbour (1967). On the Geometrical Interpretation of the Theory of Gravitation in Flat Space. Zeitschrift für Physik, 203:82-90.

Ne'eman, Y. (1980). Gravity, Groups, and Gauges. In Held (1980). 
O'Raifeartaigh, L. (1995). The Dawning of Gauge Theory. Princeton University Press, Princeton.

O'Raifeartaigh, L. and N. Straumann (2000). Gauge Theory: Historical Origins and Some Modern Developments. Reviews of Modern Physics, 72(1):1-23.

Quine, W. V. (1960). Word and Object. The MIT Press, Cambridge, Mass.

Quine, W. V. (1975). On Empirically Equivalent Systems of the World. Erkenntnis, 9:313-328.

Quine, W. V. (1990). Three Indeterminacies. In Barrett, R. B. and R. F. Gibson, eds.: Perspectives on Quine. Blackwell, Oxford.

Sciama, D. W. (1962). On the Analogy between Charge and Spin in General Relativity. In Recent Developments in General Relativity. Pergamon Press, Oxford.

Sexl, R. U. (1983). Die Hohlwelttheorie. Der mathematische und naturwissenschaftliche Unterricht, 36(8):453-460.

ThIRrIng, W. (1959). Lorentz-invariante Gravitationstheorien. Fortschritte der Physik, 7:79101.

Thirring, W. (1961). An Alternative Approach to the Theory of Gravitation. Annals of Physics, $16: 96-117$.

Trautman, A. (1980). Fiber Bundles, Gauge Fields, and Gravitation. In Held (1980).

Utiyama, R. (1956). Invariant Theoretical Interpretation of Interaction. Physical Review, 101(5):1597-1607.

Utiyama, R. (1980). Introduction to the Theory of General Gauge Fields. Progress of Theoretical Physics, 64(6):2207-2221.

van Fraassen, B. C. (1980). The Scientific Image. Clarendon Press, Oxford.

Weinstein, S. (1999). Gravity and Gauge Theory. Philosophy of Science, 66(Proceedings):S146-S155.

WeYL, H. (1929). Elektron und Gravitation. Zeitschrift für Physik, pp. 330-352. 